in the atherosclerotic vessel is that $\mathrm{CD}^{+} \mathrm{T}$ cells are activated locally in the vessel by plaque derived antigens. To determine if $\mathrm{T}$ cell priming can occur locally in the aorta, we first identified nave $\mathrm{T}$ cells in the aorta of WT and OT-II mice (transgenic mice with $\mathrm{CD}^{+} \mathrm{T}$ cells with a TCR specific for chicken ovalbumin 323-339 peptide in the context of $\mathrm{I}_{-} \mathrm{A}^{\mathrm{b}}$ ) and then challenged OT-II mice with ovalbumin while blocking lymphocyte trafficking/egress and extravasation. These experiments revealed that nave $\mathrm{T}$ cells could not only be primed directly in the vessel wall but that the kinetics and respective magnitude of $\mathrm{T}$ cell proliferation to antigen was comparable with aortic draining lymph nodes and spleen, supporting a role for the vessel as a seat of $\mathrm{T}$ cell priming. Together this data illustrates that the aorta can support antigen presentation and that $\mathrm{CD}^{+}{ }^{+} \mathrm{T}$ cells in the atherosclerotic aorta develop a more activated phenotype associated with Th1 cytokine expression, cytokines known for promoting macrophage activation and plaque instability. Most importantly, we demonstrate that the aorta has the capacity to mimic lymphoid tissue in being able to support local antigen driven proliferation of $\mathrm{CD}^{+} \mathrm{T}$ cells.

\section{NOX COMPARTMENTALIZATION AND PROTEIN OXIDATION IN VASCULAR SMOOTH MUSCLE CELLS - IMPLICATIONS IN VASCULAR DYSFUNCTION IN HYPERTENSION}

Livia de Lucca Camargo*, Augusto Cesar Montezano, Sofia Tsiropoulou, Adam Harvey, Katie Hood, Zhenbo Cao, Richard Burchmore, Neil Bulleid, Rhian Touyz. University of Glasgow

\subsection{6/heartjnl-2017-311726.179}

NADPH oxidases (Noxs) are a major source of reactive oxygen species (ROS) in vascular cells. ROS are important signalling molecules with diverse actions. Mechanisms underlying differential ROS effects may relate, in part, to subcellular localization and Nox isoform specificity. We investigated the compartmentalization of Noxs and ROS in vascular smooth muscle cells (VSMC) and questioned whether these phenomena are altered in hypertension. VSMCs isolated from mesenteric arteries of Wistar-Kyoto (WKY) and stroke-prone spontaneously hypertensive rats (SHRSP) were studied. Subcellular compartmentalization of Noxs was evaluated by immunoblotting after organelle fractionation. ROS levels were measured by chemiluminescence $\left(\mathrm{O}_{2}^{-}\right)$and amplex red $\left(\mathrm{H}_{2} \mathrm{O}_{2}\right)$ in the absence or presence of of ML171 (Nox1 inhibitor), GKT136901 (Nox1/4 inhibitor), mito-tempol (mitochondrialtargeted antioxidant) and 4-PBA (ER stress inhibitor). Protein oxidation was assessed using the fluorescent probe DCP-Rho1 for protein sulfenylation and the oxyblot assay for protein carbonylation. Oxidation of protein tyrosine phosphatases (PTP) was evaluated by immunoblotting and Peroxiredoxin (Prx) oxidation was assessed by one-dimensional isoelectric focusing. Vascular reactivity was assessed by myography \pm DTT (reducing agent) and peroxiredoxin inhibitor (Conoidin A). Expression of Nox1, Nox 2 and Nox4 was greater in total cell homogenates from SHRSP versus WKY. Nox isoforms were detected in plasma membrane, ER and nucleus in both strains, but not in the mitochondria. Basal ROS generation was increased in SHRSP cells. In WKY only Nox1 inhibition decreased Ang II-induced ROS generation. Inhibition of Nox1 and Nox4 decreased basal and Ang II-induced ROS in SHRSP.
Additionally, mito-tempol and 4-PBA reduced basal ROS generation in SHRSP. Analysis of protein oxidation revealed increased protein carbonylation and PTP oxidation in SHRSP. Furthermore, oxidation of the antioxidant enzymes Prxs was increased in SHRSP. Prx2, localised in the cytosol, and mitochondrial Prx3 were more oxidised in SHRSP cells than WKY cells. Noradrenaline-induced vascular contraction was reduced by DTT and Conoidin A. Our data demonstrate that Noxs are expressed in an organelle-specific manner, with Nox1,2,4 present in plasma membrane, ER and nucleus, but not in mitochondria. In SHRSP VSMCs Nox expression, ROS generation and protein oxidation are increased. Inhibition of oxidation attenuated vascular reactivity. These findings suggest an important role for Nox1/4 in oxidative stress and post-translational modification of proteins, processes that may play an important role in vascular dysfunction in hypertension.

\section{SYNDECAN-1 IS INVOLVED IN OSTEOPROTEGERIN- INDUCED VASCULAR DYSFUNCTION}

Augusto Montezano*, Rheure A Lopes, Karla Neves, Delyth Graham, Rhian M Touyz. University of Glasgow

\subsection{6/heartjnl-2017-311726.180}

Osteoprotegerin (OPG), an inhibitor of vascular calcification, has pleiotropic vascular effects independently of its actions on calcification. OPG has been associated with vascular inflammation and remodelling and may be important in cardiovascular disease where OPG levels may be elevated. Molecular mechanisms and functional consequences of OPG stimulation in the vasculature are unclear. We propose that syndecan-1, a membrane glycoprotein, may be important and that reactive oxygen species (ROS) play a role in OPG signalling. Vascular reactivity of resistance arteries from WKY rats was studied by wire myography in the presence or absence of OPG $(50 \mathrm{ng} /$ $\mathrm{mL}$ ) and/or synstatin (SSNT $-10^{-6} \mathrm{M}-$ syndecan-1 inhibitor). Rat endothelial cells (EC) and vascular smooth muscle cells (VSMC) were studied. Levels of ROS were measured by chemiluminescence, Amplex Red $\left(\mathrm{H}_{2} \mathrm{O}_{2}\right)$ and ELISA (nitrotyrosine; peroxynitrite - $\mathrm{ONOO}^{-}$). Protein oxidation and levels were measured by immunoblotting. Exposure of resistance arteries to OPG induced endothelial (decreased relaxation to acetylcholine) and VSMC (decreased relaxation to sodium nitroprusside - SNP) dysfunction, as well as, increased contraction to phenylephrine. All responses were blocked by SSNT, N-acetylcisteine (antioxidant) and ML171 (Nox inhibitor). In EC, OPG-induced ROS production (240 $\pm 46.1 \%$ increase $v s$. veh, $\mathrm{p}<0.05)$ was blocked by SSNT. OPG decreased $\mathrm{H}_{2} \mathrm{O}_{2}$ production/release $(61 \pm 5.4 \%$ vs. veh) and increased eNOS Thr 495 phosphorylation (inhibitory site) $(100 \pm 24 \%$ vs. veh, $\mathrm{p}<0.05)$. In VSMC, OPG increased $\mathrm{H}_{2} \mathrm{O}_{2}$ $(69 \pm 3 \%)$ and ONOO $^{-}(43 \pm 12 \%)$ levels, protein oxidation (61 $\pm 15 \%)$, Rho kinase $(200 \pm 39 \%)$ and myosin light chain activation $(55 \pm 3 \%)$ (all $v s$. veh, $\mathrm{p}<0.05)$. Increase in OPGinduced $\mathrm{ONOO}^{-}$levels was exacerbated by SNP $(130 \pm 16 \%$ vs. veh, $\mathrm{p}<0.05)$, a nitric oxide donor. In conclusion, vascular dysfunction elicited by OPG is mediated by syndecan- 1 and ROS. Whether syndecan-1 also impacts on OPG-sensitive calcification is unclear. Our data identify a novel molecular mechanism through syndecan- $1 /$ ROS that may underlie injurious effects of OPG. 